\title{
Quantification of Myocardial Perfusion Reserve Using Dynamic SPECT Imaging in Humans: A Feasibility Study
}

\author{
Simona Ben-Haim ${ }^{1-3}$, Venkatesh L. Murthy ${ }^{4,5}$, Christopher Breault ${ }^{6}$, Rayjanah Allie ${ }^{1}$, Arkadiusz Sitek ${ }^{6}$, Nathaniel Roth ${ }^{7}$, \\ Jolene Fantony ${ }^{6}$, Stephen C. Moore ${ }^{6}$, Mi-Ae Park ${ }^{6}$, Marie Kijewski ${ }^{6}$, Athar Haroon ${ }^{1}$, Piotr Slomka ${ }^{8}$, Kjell Erlandsson ${ }^{2}$, \\ Rafael Baavour ${ }^{7}$, Yoel Zilberstien ${ }^{7}$, Jamshed Bomanji ${ }^{1-3}$, and Marcelo F. Di Carli ${ }^{5,6}$ \\ ${ }^{1}$ Institute of Nuclear Medicine, University College London Hospitals, NHS Trust, London, United Kingdom; ${ }^{2}$ University College \\ London, London, United Kingdom; ${ }^{3}$ Institute of Nuclear Medicine, Chaim Sheba Medical Center, Tel Hashomer, Israel; ${ }^{4}$ Division of \\ Cardiovascular Medicine, Department of Internal Medicine, and Divisions of Nuclear Medicine and Cardiothoracic Imaging, \\ Department of Radiology, University of Michigan, Ann Arbor, Michigan; ${ }^{5}$ Noninvasive Cardiovascular Imaging Program, \\ Departments of Medicine and Radiology, Brigham and Women's Hospital, Harvard Medical School, Boston, Massachusetts; \\ ${ }^{6}$ Division of Nuclear Medicine and Molecular Imaging, Department of Radiology, Brigham and Women's Hospital, Harvard Medical \\ School, Boston, Massachusetts; ${ }^{7}$ Spectrum-Dynamics, Caesarea, Israel; and ${ }^{8}$ Departments of Imaging and Medicine and Cedars- \\ Sinai Heart Institute, Cedars-Sinai Medical Center, Los Angeles, California
}

Myocardial perfusion imaging (MPI) is well established in the diagnosis and workup of patients with known or suspected coronary artery disease (CAD); however, it can underestimate the extent of obstructive CAD. Quantification of myocardial perfusion reserve with PET can assist in the diagnosis of multivessel CAD. We evaluated the feasibility of dynamic tomographic SPECT imaging and quantification of a retention index to describe global and regional myocardial perfusion reserve using a dedicated solid-state cardiac camera. Methods: Ninety-five consecutive patients $(64$ men and 31 women; median age, $67 \mathrm{y})$ underwent dynamic SPECT imaging with $99 \mathrm{mTc}$-sestamibi at rest and at peak vasodilator stress, followed by standard gated MPI. The dynamic images were reconstructed into 60-70 frames, 3-6 s/frame, using ordered-subsets expectation maximization with 4 iterations and 32 subsets. Factor analysis was used to estimate blood-pool time-activity curves, used as input functions in a 2-compartment kinetic model. $K_{1}$ values ( ${ }^{99 \mathrm{~m} T c-s e s t a m i b i}$ uptake) were calculated for the stress and rest images, and $K_{2}$ values $(99 \mathrm{~m}$ Tc-sestamibi washout) were set to zero. Myocardial perfusion reserve (MPR) index was calculated as the ratio of the stress and rest $K_{1}$ values. Standard MPI was evaluated semiquantitatively, and total perfusion deficit (TPD) of at least $5 \%$ was defined as abnormal. Results: Global MPR index was higher in patients with normal MPI $(n=51)$ than in patients with abnormal MPI (1.61 [interquartile range (IQR), 1.33-2.03] vs. 1.27 [IQR, 1.12-1.61], $P=0.0002$ ). By multivariable regression analysis, global MPR index was associated with global stress TPD, age, and smoking. Regional MPR index was associated with the same variables and with regional stress TPD. Sixteen patients undergoing invasive coronary angiography had 20 vessels with stenosis of at least $50 \%$. The MPR index was 1.11 (IQR, 1.01-1.21) versus 1.30 (IQR, 1.12-1.67) in territories supplied by obstructed and

Received Jun. 6, 2012; revision accepted Jan. 3, 2013.

For correspondence or reprints contact: Simona Ben-Haim, Institute of Nuclear Medicine, Chaim Sheba Medical Center, Tel Hashomer, Israel 52621. E-mail: simona.ben-haim@sheba.health.gov.il

Published online Apr. 11, 2013.

COPYRIGHT $\odot 2013$ by the Society of Nuclear Medicine and Molecular Imaging, Inc. nonobstructed arteries, respectively $(P=0.02)$. MPR index showed a stepwise reduction with increasing extent of obstructive CAD $(P=0.02)$. Conclusion: Dynamic tomographic imaging and quantification of a retention index describing global and regional perfusion reserve are feasible using a solid-state camera. Preliminary results show that the MPR index is lower in patients with perfusion defects and in regions supplied by obstructed coronary arteries. Further studies are needed to establish the clinical role of this technique as an aid to semiquantitative analysis of MPI.

Key Words: dynamic SPECT; myocardial perfusion imaging; quantification; solid-state camera; coronary artery disease

J Nucl Med 2013; 54:873-879

DOI: 10.2967/jnumed.112.109652

$\mathbf{M}$ yocardial perfusion imaging (MPI) is an established, valuable tool to evaluate patients with known or suspected coronary artery disease (CAD) for the purpose of diagnosing flow-limiting disease (1), stratifying risk (2), and managing patients (3). Although the standard semiquantitative approach remains a sensitive means for diagnosing or ruling out the presence of obstructive CAD in individual patients, it often reveals only the coronary territory subtended by the vessel with the most severely flow-limiting stenosis, underestimating the extent of disease elsewhere in the coronary tree. This limitation is due to the fact that coronary vasodilator reserve is diffusely abnormal in patients with extensive CAD, leading to the so-called balanced reduction in myocardial perfusion, reduced flow heterogeneity, and underestimation of the extent of underlying obstructive CAD (4). Absolute quantification of myocardial flow reserve has been proposed as a potential approach to overcoming this limitation. Indeed, small proof-of-concept studies with PET $(5,6)$ have shown that the use of quantitative analysis of myocardial flow reserve (the ratio of peak 
myocardial blood flow to resting myocardial blood flow) can improve the diagnostic accuracy of MPI.

Quantification of tomographic myocardial perfusion from dynamic PET images is well established (7-9). Previous studies have suggested that it is also possible to quantify myocardial perfusion reserve using dynamic planar scintigraphy and ${ }^{99 \mathrm{~m}} \mathrm{Tc}$ tracers in humans $(10-15)$ or dynamic SPECT and ${ }^{201} \mathrm{Tl}$ in animals (16-18). Although these studies provided initial proof of concept, they also highlighted the limitations of conventional SPECT systems for the dynamic collection of tomographic data necessary to quantify rapid changes in radiotracer concentration. In addition, during rapid image acquisition, mechanical and patient safety constraints can limit the detector orbit to circular paths at increased distances from the patient, resulting in degraded spatial resolution. This is compounded by the relatively limited temporal resolution of conventional scintillation crystals for collecting an adequate number of counts during rapidly changing tracer concentrations. Thus, our objective was to determine the feasibility of dynamic tomographic imaging for quantification of regional and global myocardial perfusion and perfusion reserve in humans using a novel camera system equipped with cadmium zinc telluride crystals, allowing increased sensitivity and spatial resolution.

\section{MATERIALS AND METHODS}

\section{Study Cohort}

Ninety-five consecutive patients with suspected or known stable CAD referred for clinical pharmacologic stress myocardial perfusion SPECT imaging were prospectively enrolled and were included in this analysis. The study was conducted at 2 clinical sites, University College London Hospitals, National Health Service Trust, London, U.K. (55 patients), and Brigham and Women's Hospital, Boston, Massachusetts (40 patients). Patients referred for exercise stress testing were excluded from participation, as were those with significant valvular heart disease and left ventricular dysfunction (left ventricular ejection fraction $<50 \%$ ). At Brigham and Women's Hospital, patients were recruited from a pool of approximately 700 patients referred for pharmacologic stress MPI during the same study period. At University College London, patients were recruited from a pool of approximately 1,000 patients referred for MPI during the study period. For each subject, information about their past medical history and coronary risk factors was collected at the time of their imaging study.

The study was approved by the local ethics committee (University College London Hospitals) and by Partners Healthcare Institutional Review Board (Brigham and Women's Hospital); all study procedures were in accordance with institutional guidelines, and a requirement for individual informed consent was waived.

\section{SPECT Imaging}

Patients were imaged using a solid-state dedicated cardiac camera (D-SPECT; Spectrum Dynamics) after an overnight fast. They refrained from caffeine and methylxanthine-containing substances and drugs for at least $24 \mathrm{~h}$ before their scans. MPI was performed at rest and at peak vasodilator stress (with adenosine in 55 patients and dipyridamole in 40 patients) using ${ }^{99 \mathrm{~m}} \mathrm{Tc}$-sestamibi as a perfusion tracer delivered via an automatic injector (Quickfit; MEDRAD) to control the quality and reproducibility of the radiotracer injection.
To enable positioning of the heart in the field of view, a test dose of $40-100 \mathrm{MBq}$ of ${ }^{99 \mathrm{~m}} \mathrm{Tc}$-sestamibi was first injected at rest for a prescan of $60 \mathrm{~s}$. Beginning with the intravenous bolus administration of the remainder of the resting ${ }^{99 \mathrm{~m}} \mathrm{Tc}$-sestamibi dose (200-800 MBq), serial 3- to 6-s images were acquired in list mode for 6 min. Routine gated resting images were acquired in list mode after dynamic imaging. With the patient positioned in the scanner, pharmacologic stress was then performed using an intravenous infusion of adenosine $(0.140 \mathrm{mg} / \mathrm{kg} / \mathrm{min}$ for $6 \mathrm{~min})$ or dipyridamole $(0.142 \mathrm{mg} / \mathrm{kg} / \mathrm{min}$ for $4 \mathrm{~min})$. At peak stress, a second dose of 99m Tc-sestamibi (720-1,375 MBq) was injected and list-mode images were recorded in the same manner, followed by gated stress imaging. Eighty-five patients underwent a 1-d rest-stress protocol, and 10 patients (body mass index $>35$ ) had a 2-d protocol. The average radiation exposure per study was $11.19 \mathrm{mSv}(19,20)$. Heart rate, blood pressure, and 12-lead electrocardiogram were recorded at baseline and every minute during and after pharmacologic stress.

Routine rest-stress gated MPI was performed for 6 min (120 projections per detector, $3 \mathrm{~s}$ per projection) and 4 min (120 projections per detector, $2 \mathrm{~s}$ per projection) at University College London Hospitals and for $6 \mathrm{~min}$ (120 projections per detector, $2 \mathrm{~s}$ per projection) and 4 min (120 projections per detector, $1 \mathrm{~s}$ per projection) at Brigham and Women's Hospital. Acquisition times had to be adjusted at the 2 sites to account for differences in the injected radiotracer activities.

\section{Quantification of Myocardial Perfusion Images}

List-mode dynamic acquisition data were reformatted and reconstructed into 60-70 frames. Images were reconstructed using ordered-subsets expectation maximization with 4 iterations and 32 subsets, using $3 \times 3$ matrix smoothing. Factor analysis was used to generate left and right ventricular blood pool time-activity curves from the reconstructed multiframe dataset, as described previously (21). These curves served as input functions in kinetic analysis. Regional dynamic curves corresponding to cardiac territories were determined from regions of interest placed in the mid-myocardial wall. Regional and global time-activity curves were fitted to a 2-compartment kinetic model, with input functions derived using factor analysis. $K_{1}$ parameter values ( ${ }^{99 \mathrm{~m}} \mathrm{Tc}$-sestamibi uptake) were calculated for the stress and rest images, and $K_{2}$ values ( ${ }^{99 \mathrm{~m}} \mathrm{Tc}$-sestamibi washout) were set to zero. The myocardial perfusion reserve (MPR) index was then calculated as the ratio of the stress and rest $K_{1}$ values (MPR index $=K_{1}$ stress $/ K_{1}$ rest).

Relative myocardial perfusion was also evaluated semiquantitatively comparing each patient's normalized polar map with a previously validated reference database for the solid-state camera (22). The total perfusion deficit (TPD) score was calculated using QPS software. A global TPD score of 5\% or greater was defined as abnormal (23). Fixed defects were defined as a TPD stress - TPD rest score of $3 \%$ or less.

The semiquantitative analyses for TPD measurements and the MPR index computations were done independently and were then combined for statistical analysis. Interreader reliability for both regional and global MPR index estimates was assessed in 10 randomly selected patients ( 5 patients from each center) by 3 independent readers per site.

\section{Invasive Coronary Angiography}

Sixteen patients underwent coronary angiography within $90 \mathrm{~d}$ of the index SPECT study with no intervening coronary event, interventional procedure, or change in symptoms. Cineangiograms of the coronary arteries were obtained in multiple projections using 
a standard technique. The angiographic criterion used to define the presence of significant CAD was a visually determined diameter stenosis of at least $50 \%$ in the left main, left anterior descending, left circumflex, or right coronary artery and its main branch vessels. Vessels supplied by patent bypass grafts were considered patent.

\section{Statistical Analysis}

Continuous data are presented as median with interquartile ranges (IQRs). Statistical significance was assessed using Wilcoxon tests, Fisher exact tests, and $\chi^{2}$ tests for continuous, dichotomous, and categoric variables, respectively. Pearson correlations were computed to assess relationships between continuous predictors and global MPR index. Two-sided $P$ values of less than 0.05 were considered statistically significant. All statistical analyses were performed using SAS 9.3 (SAS Institute Inc.).

To evaluate the independent predictors of global MPR index, forward-stepwise linear regression was performed with an entry criterion of $P \leq 0.15$ and a removal criterion of $P>0.05$. Independent predictors of regional MPR index among age, body mass index, sex, diabetes, hypertension, dyslipidemia, smoking, prior CAD history, symptoms, relative MPI findings, and stress and rest left ventricular ejection fraction were identified using generalized linear mixed models with per-patient random intercepts. Predictor variables were added to the model in a forward-stepwise manner, analogous to the procedure used for global MPR index.

The interreader reliability was assessed with type 1 intraclass correlation coefficients, which were 0.94 (95\% CI, 0.85-0.98) for global MPR index and 0.88 (95\% CI, 0.74-0.97) for regional MPR index.

\section{RESULTS}

\section{Characteristics of the Study Cohort}

Table 1 summarizes the characteristics of the study population. One third of patients in the study were women. Forty-eight $(50.5 \%)$ patients had a body mass index of 30 or more. Dyslipidemia and hypertension were highly prevalent in the study cohort (60\% and $69 \%$, respectively), whereas diabetes was present in $37 \%$ of the patients. By semiquantitative analysis, 51 patients had normal scan results and 44 had abnormal results (19 patients had fixed perfusion defects, and 25 had reversible defects).

\section{Tomographic Dynamic SPECT Imaging}

Figure 1 shows a typical example of sequential images for a mid-ventricular short-axis slice after the intravenous injection of ${ }^{99 \mathrm{~m}} \mathrm{Tc}$-sestamibi during vasodilator stress, and their corresponding RV and LV blood pool (input function) and tissue time-activity curves. The images demonstrate initial visualization of the RV and LV blood pools, and subsequent gradual accumulation of the radiotracer in myocardial tissue over time. Despite the short dynamic frames (6 s) and the lack of postreconstruction filtering, high-quality images were obtained, reflecting the high sensitivity and resolution of the imaging system and the robustness of the dynamic imaging approach.

\section{Quantitative Analysis of Kinetic SPECT Data}

Per-Patient Analysis. Table 2 summarizes the global and regional estimates of myocardial perfusion from the kinetic
TABLE 1

Clinical Characteristics of Study Patients

\begin{tabular}{ll}
\hline \multicolumn{1}{c}{ Characteristic } & Data \\
\hline Demographics & $67(56-74)$ \\
\hline Age $(y)$ & $31(33 \%)$ \\
Female sex & $69(86 \%)$ \\
\hline Hypertension & $30(25.6-33.8)$ \\
Body mass index & \\
Coronary risk factors & $60(63 \%)$ \\
Dyslipidemia & $35(37 \%)$ \\
Diabetes & $35(36 \%)$ \\
Family history of CAD & $30(32 \%)$ \\
\hline Tobacco use & \\
Medical history & $21(22 \%)$ \\
\hline Prior myocardial infarction & $21(22 \%)$ \\
\hline Prior coronary artery bypass graft surgery & $30(32 \%)$ \\
Prior percutaneous coronary intervention & \\
Symptom status & $56(65 \%)$ \\
Chest pain & $42(44 \%)$ \\
Angina & $18(19 \%)$ \\
Nonanginal chest pain & $33(23 \%)$ \\
Dyspnea & \\
SPECT data & $63(53-69)$ \\
Left ventricular ejection fraction & $4(2-8)$ \\
Global stress TPD & $51(54 \%)$ \\
Normal scan & \\
\hline
\end{tabular}

Data are median followed by IQR in parentheses, or $n$ followed by percentage in parentheses $(n=95)$.

analysis of the SPECT images. As expected, global MPR index was higher in patients with normal scans than in those with abnormal myocardial perfusion by semiquantitative analysis (1.61 [IQR, 1.33-2.03] vs. 1.27 [IQR, 1.121.61], $P=0.0002$, Fig. 2). Multivariable regression analysis for global MPR index showed statistically significant associations with global stress TPD $(P=0.001)$ (Fig. 3), age $(P=0.008)$, and smoking history $(P=0.03)$. Left ventricular ejection fraction was not a significant predictor, perhaps because it was normal in nearly all the patients. There were no statistically significant differences between the MPR index values of men and women (1.45 [IQR, $1.22-1.76]$ vs. 1.58 [IQR, 1.27-1.87], $P=0.38$ ) or between patients who had pharmacologic stress with adenosine or dipyridamole (1.35 [IQR, 1.17-1.75] vs. 1.49 [IQR, 1.301.84 ], $P=0.22$ ). We did not observe any systematic difference between the input function obtained with adenosine or dipyridamole.

Per-Vessel Analysis. Table 2 summarizes the regional estimates of MPR index for the study cohort. MPR index was lower in territories with abnormal TPD $(\geq 5 \%)$ than in those with normal TPD (1.27 [IQR: 1.10-1.54] vs. 1.45 [IQR: 1.20-1.83], $P=0.003)$. Multivariable regression analysis for regional MPR index also showed statistically significant associations with regional stress TPD $(P=$ $0.0502)$, global stress TPD $(P=0.002)$, age $(P=0.01)$, and smoking history $(P=0.04)$. 


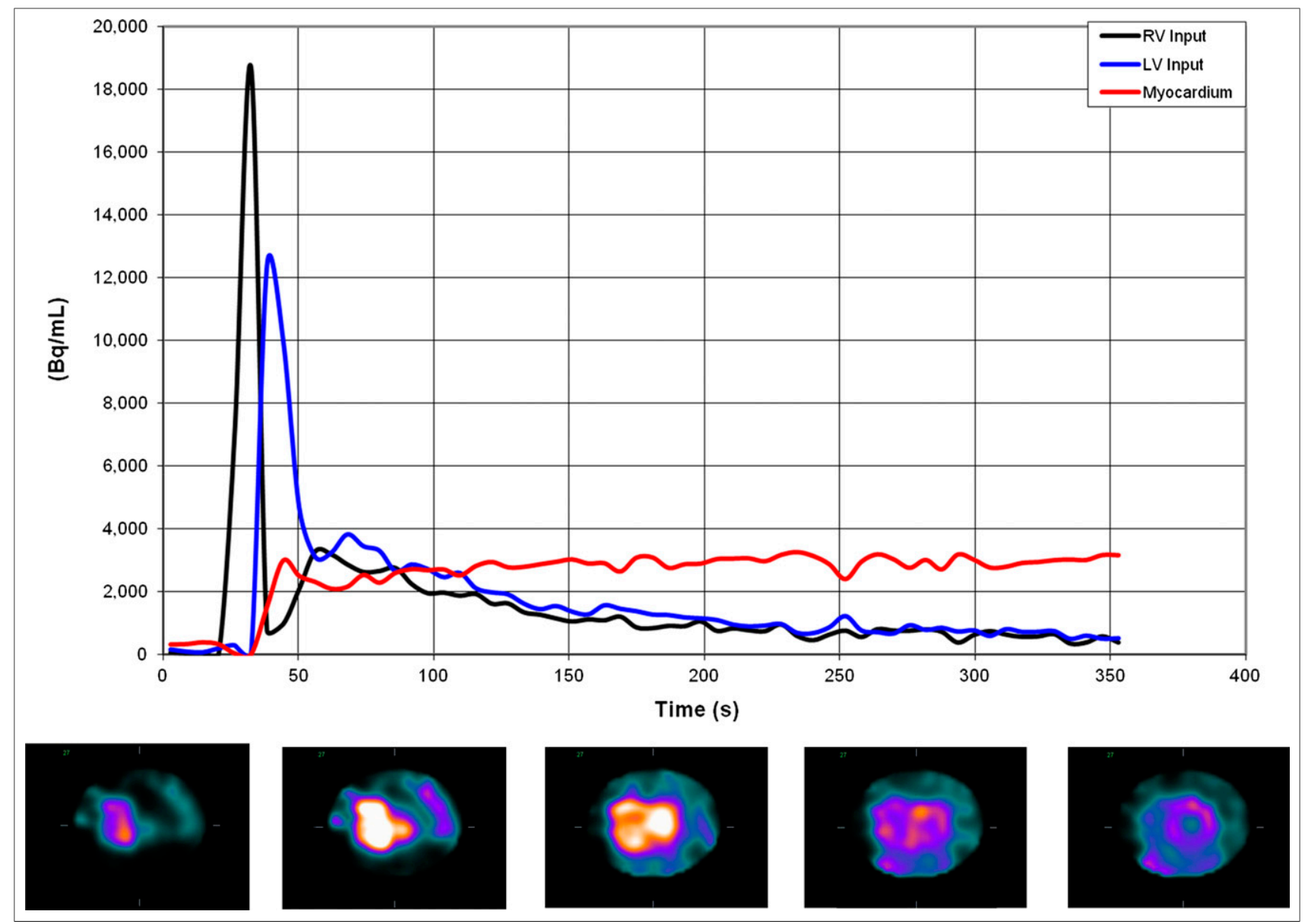

FIGURE 1. Example dynamic acquisition results. Right and left ventricular blood pool and myocardial tissue time-activity curves (upper panel). Corresponding 6-s images, midventricular short-axis slice (lower panel). RV = right ventricle; LV = left ventricle.

\section{Subgroup Analysis of Patients with Invasive Coronary Angiography}

Among the 16 patients undergoing coronary angiography, 4 had 1-vessel disease, 5 had 2-vessel disease, and 2 had 3-vessel disease. Overall, there were 20 vessels with at least $50 \%$ stenosis and 28 vessels with nonobstructive atherosclerosis.

MPR index was lower in territories supplied by coronary arteries with at least $50 \%$ stenosis than in those with nonobstructive disease (1.11 [IQR, 1.01-121] vs. 1.30 [IQR, 1.12-1.67], $P=0.002$, Fig. 4). Global MPR index showed a stepwise decrease with increasing extent of obstructive CAD on angiography $(P=0.02$, Fig. 5$)$. In the 7 patients with 2- or 3-vessel disease, MPR index was 1.08 (IQR, 0.95-1.23), compared with 1.18 (IQR, 1.12-1.51) for the 4 patients with 1 -vessel disease and 1.58 (IQR, 1.30-1.75) for the 5 patients with no obstructive lesions.

Figure 6 shows an example of the potential added diagnostic value of MPR index in a patient with 2-vessel disease and no significant perfusion abnormalities on ${ }^{99 m}$ Tc-sestamibi MPI.

\section{DISCUSSION}

Myocardial perfusion SPECT is a robust and widely used imaging tool in the evaluation of patients with known or suspected CAD. Although its diagnostic and prognostic value is well established, there is increasing recognition that this technique often underestimates the extent of obstructive CAD in patients with severe multivessel disease

TABLE 2

Global and Regional Myocardial Perfusion Estimates from Dynamic SPECT

\begin{tabular}{lc}
\hline MPR index $\left(K_{1}\right.$ stress $/ K_{1}$ rest) & Data \\
\hline $\begin{array}{l}\text { Global estimate (left ventricular) } \\
\text { Regional estimate }\end{array}$ & $1.46(1.22-1.79)$ \\
$\quad \begin{array}{l}\text { Left anterior descending coronary } \\
\text { artery territory }\end{array}$ & $1.4(1.17-1.75)$ \\
$\quad \begin{array}{l}\text { Left circumflex coronary artery } \\
\text { territory }\end{array}$ & $1.4(1.15-1.74)$ \\
$\quad$ Right coronary artery territory & $1.53(1.22-2.01)$
\end{tabular}

Data are median followed by IQR in parentheses $(n=95)$. 


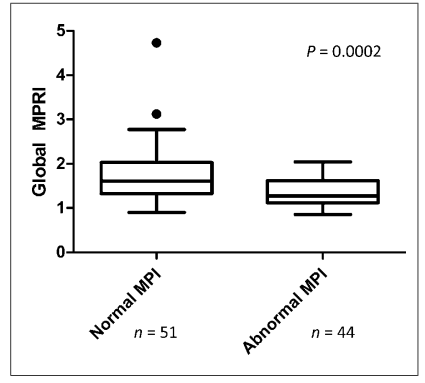

FIGURE 2. Global MPR index in normal and abnormal MPI. Filled circles represent data points outside error bars.

$(4,6)$. In addition, a normal MPI result does not necessarily identify truly low-risk subgroups among high-risk cohorts $(24,25)$. Recent evidence suggests that quantitative estimates of myocardial perfusion reserve may be useful imaging markers of clinical risk that can help identify low-risk individuals, thereby enhancing the value of MPI for risk stratification $(26,27)$. These measurements are feasible in clinical practice using PET $(6-8,28-32)$ but have not been applied to patients with SPECT.

Nonetheless, several studies have demonstrated that it is possible to obtain absolute indices of regional myocardial perfusion using dynamic SPECT in experimental animal models $(16-18)$. The use of dynamic SPECT with ${ }^{99 m} \mathrm{Tc}-$ teboroxime as the flow tracer combined with compartmental modeling to compute absolute myocardial blood flow has been previously reported (33-35). The translation of this approach to the clinical setting has been difficult because of limitations in the detector sensitivity and temporal resolution of conventional SPECT systems, which are critical for quantification of rapid changes in radiotracer activity in the blood pool and myocardial tissue. As a result, prior studies attempting to quantify myocardial perfusion reserve with ${ }^{99 \mathrm{~m}} \mathrm{Tc}$-based tracers relied on planar imaging $(11-15,36)$. The procedure is based on the microsphere method, that is, the assumption that myocardial uptake of the radiotracer is proportional to myocardial blood flow. First-pass planar imaging is performed, and right pulmonary artery counts are used as the arterial input function. Indices of myocardial perfusion at stress and rest are obtained by dividing myocardial counts by the input function. An index of myocardial perfusion reserve can then be obtained by dividing myocardial perfusion values for stress and rest. This method has several disadvantages. First, corrections for attenuation, scatter, and partial-volume effects are not possible. Second, estimates of regional and segmental blood flow are challenging because of overlapping structures. Nonetheless, estimates obtained with this technique demonstrated a reasonable correlation with measurements obtained using intravascular Doppler ultrasound (12) and ${ }^{15} \mathrm{O}_{-} \mathrm{H}_{2} \mathrm{O}$ PET (11), with systematic underestimation of flow measurements at high flow rates. Daniele et al. (15) have recently demonstrated that estimates of CFR, obtained using this method in a group of 99 patients, provided incremental prognostic information over the information from clinical and perfusion data, mainly in patients with

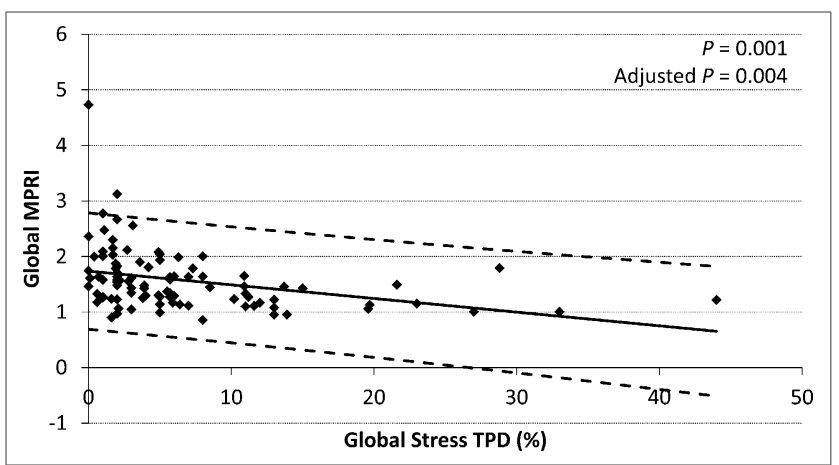

FIGURE 3. Correlation of global MPR index (MPRI) and global stress TPD.

normal MPI results. In these patients, a reduced flow reserve index was associated with higher rates of major adverse cardiac events (5.2\% vs. 0.7\%, 5.8-y mean follow-up).

To our knowledge, this is the first report evaluating the feasibility of dynamic tomographic imaging and quantification of regional and global myocardial perfusion and perfusion reserve in humans using a novel camera system equipped with cadmium zinc telluride crystals and ${ }^{99 \mathrm{~m} T c-}$ sestamibi. Our findings demonstrate that dynamic SPECT imaging coupled with kinetic analysis of myocardial radiotracer concentration in human myocardium is feasible and reproducible. We showed that regions of abnormal perfusion by semiquantitative analysis display appropriate reductions in myocardial perfusion reserve compared with that of visually normal remote myocardium. We also showed preliminary evidence that global measures of MPR index obtained with ${ }^{99 \mathrm{~m}} \mathrm{Tc}$ agents and SPECT correlate inversely with the extent of obstructive CAD on coronary angiography. Among patients with correlative invasive coronary angiography, MPR index values were significantly lower in territories subtended by vessels with severe stenoses than in territories supplied by nonobstructed coronary arteries. Furthermore, there was a significant stepwise reduction in global MPR index values with increasing extent of obstructive CAD. Given the growing importance of absolute quantification of myocardial perfusion and perfusion reserve in diagnosis and, especially, risk prediction, we believe that our findings may have important implications to bolster the role of SPECT in the evaluation of patients with known or suspected CAD.

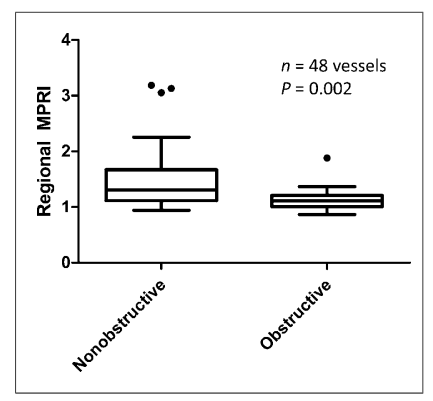

FIGURE 4. Regional MPR index (MPRI) by coronary angiography findings. Obstructive $=$ $\geq 50 \%$. 
FIGURE 5. Global MPR index by angiographic disease extent. VD $=$ vessel disease $(\geq 50 \%)$.

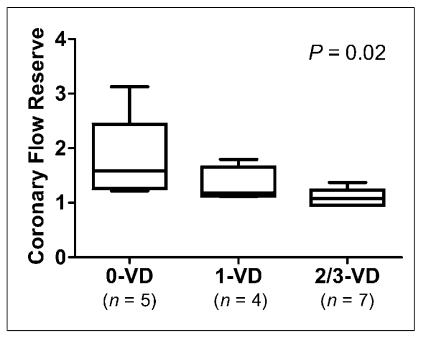

The relatively small difference in MPR index between patients with normal and abnormal relative MPI may be due to several reasons. First, the MPR index is a retention index that does not model radiotracer extraction, and the somewhat limited range is related, at least in part, to the roll-off in the extraction of sestamibi at higher flow rates. In fact, the use of a similar retention index with ${ }^{82} \mathrm{Rb}$ PET yielded a similarly narrow range of MPR index (6). Second, most of our patients have coronary risk factors including dyslipidemia, hypertension, and diabetes, and about one third of the patients had known CAD. These factors have been shown in prior PET studies to be associated with reduced CFR even in the absence of focal epicardial stenosis, reflecting both diffuse coronary atherosclerosis and microvascular dysfunction. Consequently, the MPR index in patients without relative MPI abnormalities is likely abnormal, thereby diminishing the observed difference between patients with and without relative MPI abnormalities.

There were some limitations to this study. ${ }^{99 \mathrm{~m}} \mathrm{Tc}$-sestamibi was used as the perfusion agent for the measurement of MPR index. Consequently, retention estimates at high flow rates may be underestimated because of the nonlinear relationship between radiotracer extraction and myocardial blood flow. Therefore further studies in larger patient cohorts may be required to define the normal limits of MPR index measurements for this tracer.
Because corrections for attenuation and scatter were not available, quantitation of myocardial blood flow in $\mathrm{mL} / \mathrm{min} / \mathrm{g}$ of myocardium could not be obtained. However, because we defined MPR index as a ratio of tracer uptake at stress and at rest, attenuation should be similar in both scans. With further hardware and software developments, absolute quantitation of flow using SPECT may soon become feasible.

The study was conducted on a novel dedicated cardiac camera with solid-state detectors, which is currently not widely used, and therefore the generalizability of this approach is limited at present.

Although the improved count sensitivity of this camera may allow dynamic studies using a lower injected dose, the impact of a lower injected dose on the accuracy and reproducibility of dynamic imaging will need to be defined in future studies.

No gold standard was available to confirm the accuracy of the MPR index measurements. However, we could demonstrate good correlations between MPR index and visual estimates of global and regional myocardial perfusion (i.e., TPD) and, in a subgroup of patients, anatomic measures of CAD severity. Unfortunately, only a few patients were referred for coronary angiography despite regional perfusion abnormalities, reflecting conservative medical management of patients with modest disease burdens. Future studies, on larger patient cohorts, comparing MPR index values with a well-established absolute perfusion technique such as PET are warranted.

\section{CONCLUSION}

Dynamic tomographic imaging and quantification of a retention index describing regional and global myocardial perfusion and perfusion reserve in humans are feasible and reproducible using a novel SPECT camera system equipped with cadmium zinc telluride crystals. SPECT measures of
FIGURE 6. Case example: 70-y-old man with body mass index of 19.5 and previous infarction. (A) Stress (top row) and rest (bottom row) ${ }^{99 m T c-s e s t a m i b i ~ M P I ~ s h o w i n g ~ n o r-~}$ mal myocardial perfusion. (B) Regional MPR index (MPRi) is low in left anterior descending (LAD) and right coronary artery (RCA) territories. Coronary angiography confirmed proximal obstruction of left anterior descending coronary artery and severe diffuse disease of right coronary artery. LCX = left circumflex artery.
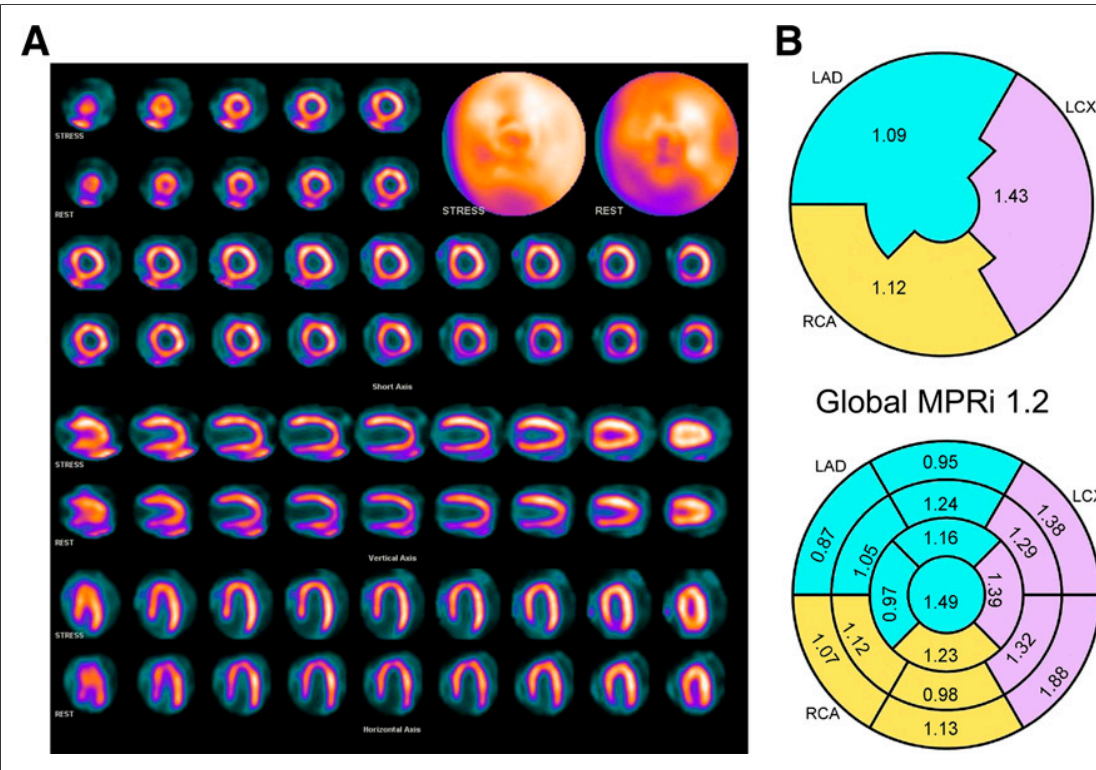

Global MPRi 1.2

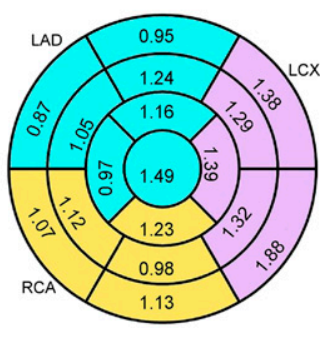


myocardial perfusion reserve are lower in patients with perfusion defects and in territories supplied by obstructed arteries and show a stepwise decrease with increasing extent of obstructive CAD. Further studies are warranted to establish the role of this technique in the clinical workup of patients with known or suspected CAD.

\section{DISCLOSURE}

The costs of publication of this article were defrayed in part by the payment of page charges. Therefore, and solely to indicate this fact, this article is hereby marked "advertisement" in accordance with 18 USC section 1734. Part of this work was undertaken at UCLH/UCL, which received a proportion of funding from the U.K. Department of Health's NIHR Biomedical Research Centres funding scheme. This study was funded in part by grants from the U.S. National Institutes of Health (RC1 HL101060-01 and T32 HL094301-01A1). Dr. Ben-Haim is a consultant for Spectrum-Dynamics. Dr. Murthy owns stock in General Electric. Dr. Piotr Slomka participates in software royalties at Cedars-Sinai Medical Center. Nathaniel Roth, Rafael Baavour, and Yoel Zilberstien are employed by SpectrumDynamics. Dr. Di Carli receives research grant support from Toshiba. No other potential conflict of interest relevant to this article was reported.

\section{REFERENCES}

1. Gibbons RJ, Chatterjee K, Daley J, et al. ACC/AHA/ACP-ASIM guidelines for the management of patients with chronic stable angina: a report of the American College of Cardiology/American Heart Association Task Force on Practice Guidelines (Committee on Management of Patients with Chronic Stable Angina). J Am Coll Cardiol. 1999;33:2092-2197.

2. Shaw LJ, Iskandrian AE. Prognostic value of gated myocardial perfusion SPECT. J Nucl Cardiol. 2004;11:171-185.

3. Hachamovitch R, Hayes S, Friedman JD, et al. Determinants of risk and its temporal variation in patients with normal stress myocardial perfusion scans: what is the warranty period of a normal scan? J Am Coll Cardiol. 2003;41:1329-1340.

4. Berman DS, Kang X, Slomka PJ, et al. Underestimation of extent of ischemia by gated SPECT myocardial perfusion imaging in patients with left main coronary artery disease. J Nucl Cardiol. 2007;14:521-528.

5. Ziadi MC, deKemp RA, Williams $\mathrm{K}$, et al. Does quantification of myocardial flow reserve using rubidium- 82 positron emission tomography facilitate detection of multivessel coronary artery disease? J Nucl Cardiol. 2012;19:670-680.

6. Parkash R, deKemp RA, Ruddy TD, et al. Potential utility of rubidium 82 PET quantification in patients with 3-vessel coronary artery disease. $\mathrm{J} \mathrm{Nucl} \mathrm{Cardiol.}$ 2004;11:440-449.

7. Tio RA, Dabeshlim A, Siebelink H-MJ, et al. Comparison between the prognostic value of left ventricular function and myocardial perfusion reserve in patients with ischemic heart disease. J Nucl Med. 2009;50:214-219.

8. Herzog BA, Husmann L, Valenta I, et al. Long-term prognostic value of ${ }^{13} \mathrm{~N}$ ammonia myocardial perfusion positron emission tomography: added value of coronary flow reserve. J Am Coll Cardiol. 2009;54:150-156.

9. Murthy VL, Naya M, Foster CR, et al. Improved cardiac risk assessment with noninvasive measures of coronary flow reserve. Circulation. 2011;124:22152224 .

10. Taki J, Fujino S, Nakajima K, et al. Tc-99m retention characteristics during pharmacological hyperemia in human myocardium: comparison with coronary flow reserve measured by Doppler flowire. J Nucl Med. 2001;42:1457-1463.

11. Ito $\mathrm{Y}$, Katoh C, Noriyasu $\mathrm{K}$, et al. Estimation of myocardial blood flow and myocardial flow reserve by $99 \mathrm{~m}$-technetium sestamibi imaging: comparison with the results of $15 \mathrm{H}_{2} \mathrm{O}$ PET. Eur J Nucl Med Mol Imaging. 2003;30:281-287.

12. Storto G, Cirillo P, Vicario ML, et al. Estimation of coronary flow reserve by Tc$99 \mathrm{~m}$ sestamibi imaging in patients with coronary artery disease: comparison with the results of intracoronary Doppler technique. J Nucl Cardiol. 2004;11:682-688.
13. Storto G, Soricelli A, Pellegrino T, Petretta M, Cuocolo A. Assessment of the arterial input function for estimation of coronary flow reserve by single photon emission computed tomography: comparison of two different approaches. Eur J Nucl Med Mol Imaging. 2009;36:2034-2041.

14. Marini C, Giusti M, Armonino R, et al. Reduced coronary flow reserve in patients with primary hyperparathyroidism: a study by G-SPECT myocardial perfusion imaging. Eur J Nucl Med Mol Imaging. 2010;37:2256-2263.

15. Daniele $\mathrm{S}$, Nappi $\mathrm{C}$, Acampa W, et al. Incremental prognostic value of coronary flow reserve assessed with single-photon emission computed tomography. J Nucl Cardiol. 2011;18:612-619.

16. Iida H, Eberl S. Quantitative assessment of regional myocardial blood flow with thallium-201 and SPECT. J Nucl Cardiol. 1998;5:313-331.

17. Christian TF, Peters K, Keck B, Allen J, Owens T, Borah B. Gated SPECT imaging to detect changes in myocardial blood flow during progressive coronary occlusion. Int J Cardiovasc Imaging. 2008;24:269-276.

18. Iida H, Eberl S, Kim KM, et al. Absolute quantitation of myocardial blood flow with ${ }^{201} \mathrm{Tl}$ and dynamic SPECT in canine: optimization and validation of kinetic modeling. Eur J Nucl Med Mol Imaging. 2008;35:896-905.

19. Stabin MG. Radiopharmaceuticals for nuclear cardiology: radiation dosimetry, uncertainties and risk. J Nucl Med. 2008;49:1555-1563.

20. Radiation dose to patients from radiopharmaceuticals (addendum to ICRP publication 53): ICRP publication 80. Ann ICRP. 1998;28.

21. Sitek A, Gullberg GT, Huesman RH. Correction for ambiguous solutions in factor analysis using a penalized least squares objective. IEEE Trans Med Imaging. 2002;21:216-225.

22. Nakazato R, Tamarappoo BK, Kang X, et al. Quantitative upright-supine highspeed SPECT myocardial perfusion imaging for detection of coronary artery disease: correlation with invasive coronary angiography. J Nucl Med. 2010;51: 1724-1731.

23. Sharir T, Slomka PJ, Hayes SW, et al. Multicenter trial of high-speed versus conventional single-photon emission computed tomography imaging. J Am Coll Cardiol. 2010;55:1965-1974.

24. Giri S, Shaw LJ, Murthy DR, et al. Impact of diabetes on the risk stratification using stress single-photon emission computed tomography myocardial perfusion imaging in patients with symptoms suggestive of coronary artery disease. Circulation. 2002;105:32-40.

25. Al-Mallah MH, Hachamovitch R, Dorbala S, Di Carli F. Incremental prognostic value of myocardial perfusion imaging in patients referred for stress singlephoton emission computed tomography with renal dysfunction. Circ Cardiovasc Imaging. 2009;2:429-436.

26. Murthy VL, Naya M, Foster CR, et al. Coronary vascular dysfunction and prognosis in patients with chronic renal disease. J Am Coll Cardiol Imaging. 2012;5:1025-1034.

27. Murthy VL, Naya M, Foster CR, et al. Association between coronary vascular dysfunction and cardiac mortality in patients with and without diabetes mellitus. Circulation. 2012;126:1858-1868.

28. Yoshinaga K, Chow BJ, deKemp RA, et al. What is the prognostic value of myocardial perfusion imaging using rubidium-82 positron emission tomography? J Am Coll Cardiol. 2006;48:1029-1039.

29. Czernin J, Muller P, Chan S, et al. Influence of age and hemodynamics on myocardial blood flow and flow reserve. Circulation. 1993;88:62-69.

30. Gould KL, Martucci JP, Goldberg DI, et al. Short-term cholesterol lowering decreases size and severity of perfusion abnormalities by positron emission tomography after dipyridamole in patients with coronary artery disease: a potential noninvasive marker of healing coronary endothelium. Circulation. 1994; 89:1530-1538.

31. Kaufmann PA, Gnecchi-Ruscone T, Di TM, Schafers KP, Luscher TF, Camici PG. Coronary heart disease in smokers: vitamin $\mathrm{C}$ restores coronary microcirculatory function. Circulation. 2000;102:1233-1238.

32. Ziadi MC, Beanlands RSB. The clinical utility of assessing myocardial blood flow using positron emission tomography. J Nucl Cardiol. 2010;17:571-581.

33. Smith AM, Gullberg GT, Christian PE, Datz FL. Kinetic modeling of teboroxime using dynamic SPECT imaging of a canine model. J Nucl Med. 1994;35:484495.

34. DiBella EVR, Khare HS, Kadrmas DJ, Gullberg GT. SPECT imaging of teboroxime during myocardial blood flow changes. IEEE Trans Nucl Sci. 2000;47: 1112-1117.

35. Di Bella EVR, Ross SG, Kadrmas DJ, et al. Compartmental modeling of technetium-99m-labeled teboroxime with dynamic single-photon-emission computed tomography: comparison with static thallium-201 in a canine model. Invest Radiol. 2001;36:178-185.

36. Sugihara H, Yonekura Y, Katoka K, Fukai D, Kitamura N, Tamiguchi Y. Estimation of coronary flow reserve with the use of dynamic SPECT images of Tc99m tetrofosmin. J Nucl Cardiol. 2001;8:575-579. 